\title{
Maternal dietary folate, folic acid and vitamin $D$ intakes during pregnancy and lactation and the risk of cows' milk allergy in the offspring
}

\author{
Jetta Tuokkola ${ }^{1,2 *}$, Päivi Luukkainen ${ }^{1}$, Minna Kaila ${ }^{3,4}$, Hanna-Mari Takkinen ${ }^{2,5}$, Sari Niinistö ${ }^{2}$, \\ Riitta Veijola ${ }^{6}$, Lauri J. Virta ${ }^{7}$, Mikael Knip ${ }^{1,8,9}$, Olli Simell ${ }^{10}$, Jorma Ilonen ${ }^{11,12}$ and Suvi M. Virtanen $^{2,5,13}$ \\ ${ }^{1}$ Children's Hospital, University of Helsinki, Helsinki University Hospital, Helsinki, OOO29 HUH, Finland \\ ${ }^{2}$ Nutrition Unit, National Institute for Health and Welfare, 00271 Helsinki, Finland \\ ${ }^{3}$ Public Health Medicine, O0014 University of Helsinki, Helsinki University Hospital, Helsinki, Finland \\ ${ }^{4}$ Department of Pediatrics, Tampere University Hospital, 33521 Tampere, Finland \\ ${ }^{5}$ School of Health Sciences, 33014 University of Tampere, Tampere, Finland \\ ${ }^{6}$ Department of Paediatrics, 90014 University of Oulu, Oulu, Finland \\ ${ }^{7}$ Research Department, Social Insurance Institution, 20720 Turku, Finland \\ ${ }^{8}$ Research Program Unit, Diabetes and Obesity, O0014 University of Helsinki, Helsinki University Hospital, Helsinki, Finland \\ ${ }^{9}$ Folkhälsan Research Institute, O0251 Helsinki, Finland \\ ${ }^{10}$ Department of Pediatrics, 20014 University of Turku, Turku, Finland \\ ${ }^{11}$ Department of Clinical Microbiology, University of Eastern Finland, 70211 Kuopio, Finland \\ ${ }^{12}$ Immunogenetics Laboratory, 20014 University of Turku, Turku, Finland \\ ${ }^{13}$ Science Center of Pirkanmaa Hospital District, 33521 Tampere, Finland
}

(Submitted 15 January 2016 - Final revision received 7 May 2016 - Accepted 23 May 2016 - First published online 28 June 2016)

\begin{abstract}
Maternal nutrient intake during pregnancy and lactation potentially influences the development of allergic diseases. Cows' milk allergy (CMA) is often the first manifestation of atopic diseases, but the impact of early nutritional influences on CMA has not been explored. The associations between maternal intakes of folate, folic acid and vitamin D during pregnancy and lactation were addressed in a prospective, populationbased birth cohort within the Finnish Type 1 Diabetes Prediction and Prevention Study. Mothers of 4921 children during pregnancy and 2940 children during lactation provided information on maternal dietary intake during the 8th month of pregnancy and the 3rd month of lactation using a detailed, validated FFQ. Information on diagnosed CMA in the offspring was obtained from a medical registry as well as queried from the parents. The Finnish food composition database was used to calculate nutrient intake. Logistic regression was applied for statistical analyses. Folate intake and folic acid and vitamin D supplement use were associated with an increased risk of CMA in the offspring, whereas vitamin D intake from foods during pregnancy was associated with a decreased risk of CMA. Thus, maternal nutrient intake during pregnancy and lactation may affect the development of CMA in offspring. Supplementation with folic acid may not be beneficial in terms of CMA development, especially in children of allergic mothers. The association between dietary supplement use and CMA risk can at least partly be explained by increased health-seeking behaviour among more educated mothers who also use more dietary supplements.
\end{abstract}

Key words: Cows' milk allergy: Lactation: Maternal diet: Nutrients: Pregnancy

Maternal nutrition during pregnancy and lactation, among other environmental factors, is suggested to have an impact on the development of allergic diseases. These early influences may be especially important in the development of cows' milk allergy (CMA), which is often the first manifestation of allergic disease. Folic acid and vitamin D have been shown to exert immunological effects, and they could potentially influence the development of allergic diseases ${ }^{(1)}$. Folic acid serves as a methyl donor and is likely to have epigenetic effects in offspring. In mice, high intake of folic acid led to the development of allergic phenotypes and systemic allergic reactions in offspring ${ }^{(2)}$. Vitamin D has several routes through which it modifies both adaptive and innate immune responses and T-helper 1/T-helper 2 (Th1/Th2) balance, and its effects could expand to development of food allergy (recently reviewed by Bozzetto et al. ${ }^{(3)}$ ). Both these nutrients have been of interest with respect to risks of other

Abbreviations: CMA, cows' milk allergy; DIPP, Finnish Type 1 Diabetes Prediction and Prevention study.

* Corresponding author: J. Tuokkola, fax +35894717 3791, email jetta.tuokkola@iki.fi 
allergic diseases, but their associations with the risk of CMA have not been previously explored.

We have previously published findings of a food-based approach studying the associations between maternal food consumption during pregnancy and lactation and subsequent CMA risk in the offspring ${ }^{(4)}$. The aims of the present study were to investigate the associations between maternal dietary intake of folate, folic acid and vitamin D during pregnancy and lactation and subsequent development of CMA in the offspring.

\section{Methods \\ Subjects}

The Finnish Type 1 Diabetes Prediction and Prevention (DIPP) study is a multidisciplinary, prospective, population-based birth cohort study $^{(5)}$. After parental informed consent, all newborn infants from the catchment areas of three university hospitals in Finland (Turku, Oulu and Tampere) were screened for human leucocyte antigen (HLA)-conferred susceptibility to type 1 diabetes from cord blood samples. Infants who carried HLA genotypes conferring high and moderate risk for type 1 diabetes ( $15 \%$ of those screened) were invited to participate in the study, excluding children with severe congenital abnormalities or diseases, or whose parents were of non-Caucasian origin or did not understand Finnish, Swedish or English. The local Ethics Committees approved the study. All families provided their written informed consent.

The DIPP Nutrition study was performed among children who were born in Oulu and Tampere university hospital areas of the DIPP study population. The present study comprised of 6288 children born between October 1997 and September 2004 and whose personal identification codes were disclosed to the study (Fig. 1). Mothers of 4921 children provided dietary data from a total of 4861 pregnancies for the pregnancy study. The lactation study was started in August 1998, collecting dietary data of the mothers who were still breast-feeding when the baby was 3 months of age. Dietary data were available for 2940 children from 2915 mothers. The numbers of mothers and children differ because of twin pregnancies. For the analysis concerning diet during breast-feeding, 120 children whose CMA had begun before completing the FFQ were omitted from the analysis, leaving us with a total of 2820 mother-child pairs for crude analysis (Fig. 1).

\section{Dietary assessment}

Maternal diet during pregnancy (8th month) and lactation (3rd month) was assessed using a validated, 181-item, semi-quantitative $\mathrm{FFQ}^{(6)}$. Mothers received the FFQ concerning
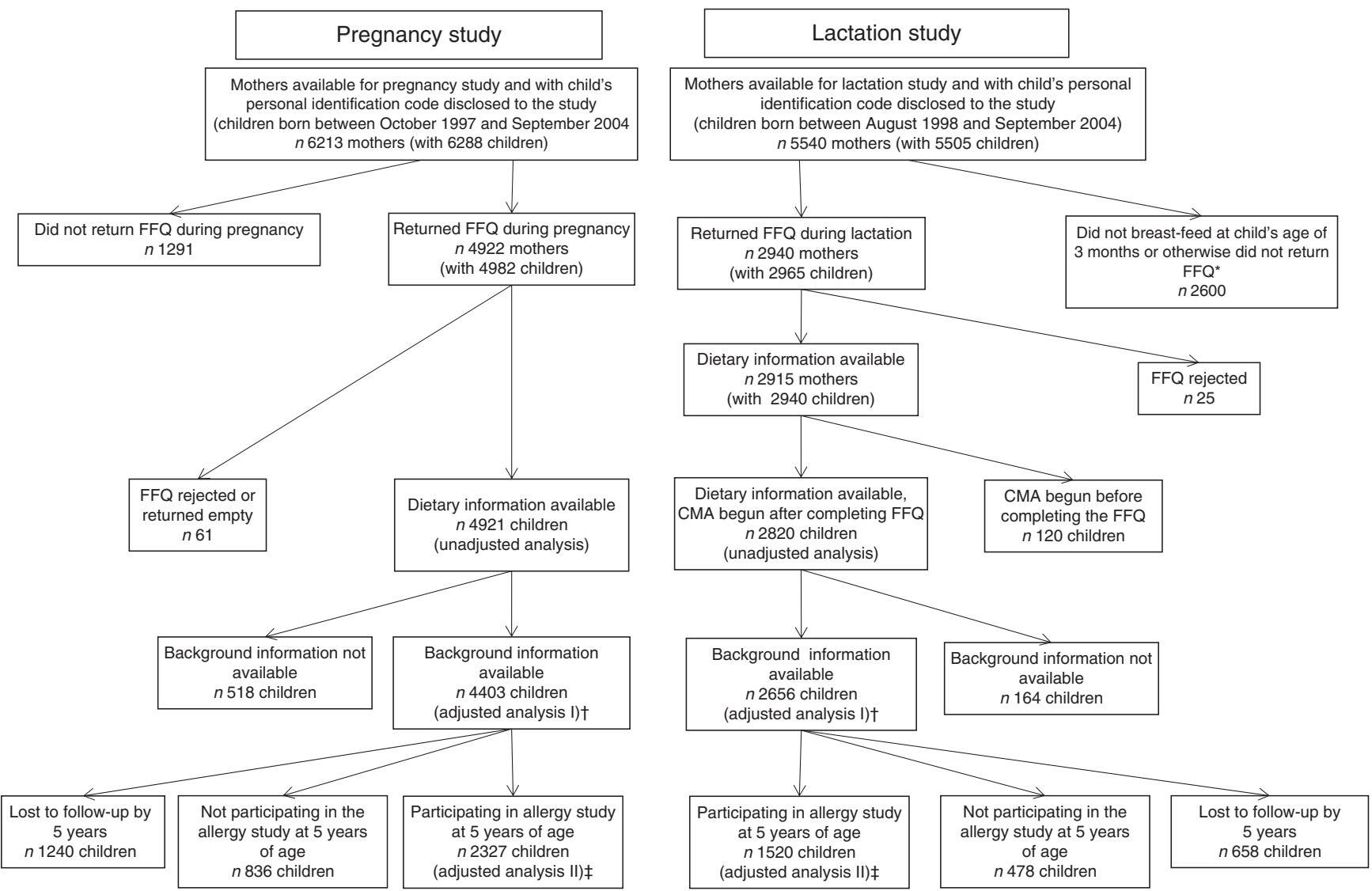

Fig. 1. Flow chart of the participants in the pregnancy and lactation studies. CMA, cows' milk allergy. ${ }^{*}$ The number of breast-fed children at the age of 3 months was 3757 - that is, $68 \%$ of the children. †Background information variables: study centre, sex, birth weight of the child, maternal age and education, maternal smoking status during pregnancy, mode of delivery, number of older siblings, duration of gestation, season of birth and length of breast-feeding. $¥$ Variables that were queried at 5 years of age: maternal asthma or allergic rhinitis, urbanity of the living environment, visits to a stable and pet keeping during the first year of life. 
the diet at the 8th month of pregnancy after delivery by mail, and it was returned at a visit to the study centre and checked by a trained study nurse when the child was 3 months old. The questionnaire concerning diet during lactation was administered at the 3-month visit and returned at the 6-month visit to the study centre. The FFQ assessed the consumption frequency of foods or food groups (not at all, number of times per day, week or month) as common serving sizes such as a glass, a plateful or decilitre. The questionnaire was specifically designed to reflect Finnish food consumption habits. Types of fat used for cooking and baking were taken into account individually. The food consumption data were double-entered. If there were ten or more missing frequencies or the form was inadequately filled in, the FFQ was rejected ( $n 61,1.2 \%$ of FFQ concerning diet during pregnancy and $n 25,0.9 \%$ of FFQ concerning diet during lactation). Daily intakes of nutrients were calculated using the Finnish national food composition database ${ }^{(7)}$ by an in-house software of the National Institute for Health and Welfare. The detailed content of the FFQ and data processing have been described elsewhere ${ }^{(6,8)}$. We used two updated versions of the database: the first version for the study years 1997-2002 and the second version for the years 2003/2004. Recipe compositions were changed from the first version to the second, in order to reflect changes in food consumption habits and changes in the food market. The changes in recipes were mainly based on food consumption information of women aged 25-44 years from the national dietary surveys FINDIET $1997^{(9)}$ and FINDIET $2002^{(10)}$. Vitamin D concentrations were systematically changed in milk and fat products because of changes in fortification in 2003 . Recording of the FFQ and the accuracy of the nutrient database of the National Public Health Institute were checked at dietary analysis. The use of dietary supplements was determined with a question in the FFQ form, asking about the type, brand name and manufacturer's name, the amount of each supplement taken per day or per week and the pregnancy weeks during which the supplements were used. The nutrient contents of the dietary supplements were obtained from the National Food Administration, the manufacturers and from the Finnish pharmacopeia for supplements registered as drugs.

\section{End points}

Information on CMA from the registers of the Social Insurance Institution, complemented with parental reports, was used as the end point. In Finland, infants who need special infant formulae for the management of diagnosed CMA (ICD-10 codes L27.2 or K52.2) are entitled to special reimbursement for costs up to 2 years of age. Information on this physician-diagnosed CMA was received from a register maintained by the Social Insurance Institution. Paediatricians have agreed on the criteria for the diagnosis (a response to an elimination diet and an open challenge; rarely, a response to an elimination diet with positive skin prick test or specific IgE; in select rare cases double-blind, placebo-controlled challenge is performed). In addition, CMA up to the age of 3 years was queried with open questions from parents at the age of 6 months and 1 and 2 years and with a structured, validated questionnaire at the age of 3 years ${ }^{(11,12)}$.

\section{Background factors}

Families were asked for information on parental vocational education, age and place of residence at recruitment. Information on pregnancy and delivery complications, gestational age, birth weight and height, earlier deliveries and maternal smoking status during pregnancy were received from the National Medical Birth Registry. Information on breast-feeding was obtained from parents at study visits. Parental atopic history, pet keeping and farm animal contacts during the child's 1st year of life were queried when the child was 5 years old with questions on parental history of asthma, allergic rhinitis and atopic eczema among the families who participated in the allergy study at the 5-year follow-up ${ }^{(13)}$.

\section{Statistical methods}

Differences in background characteristics between cases and controls and between mothers with and without allergic history were assessed using $\chi^{2}$ or Fisher's exact tests. Wilcoxon's signedrank test was used for pairwise comparison for difference between intakes during pregnancy and lactation. The Mann-Whitney $U$ test was used to assess differences in nutrient intakes between mothers with and without allergic history. Logistic regression was applied to study the associations between maternal nutrient intakes and the risk of CMA in the offspring. The possible dependence among siblings was accounted for by using the generalised estimating equations with the sandwich estimator of variance to estimate regression coefficients in the logistic regression analysis ${ }^{(14)}$. The dietary nutrient intakes were adjusted for energy intake by the residual method ${ }^{(15)}$ after logarithmic transformation; supplemental intake was not adjusted for energy. The total intake of each nutrient was calculated as the sum of intake from foods and supplements. Total and dietary nutrient intake variables were used as continuous explanatory variables in the analysis. Nutrient intakes from supplements were categorised into users and nonusers. The sex and birth weight of the child, maternal age and education, maternal smoking status during pregnancy, duration of gestation, type of delivery, number of older siblings, season of birth, length of breast-feeding, atopic family history, study centre, urbanity of the living environment (urban/semi-rural/rural), visits to a stable and pet keeping during the 1st year of life were adjusted for in the analysis. Those whose CMA diagnosis was confirmed before completing the FFQ during lactation were excluded from the analysis concerning diet during lactation. Interaction between maternal allergic history (mother has asthma or allergic rhinitis) and nutrient intakes was tested, and analyses were carried out separately for allergic and non-allergic mothers if the interaction was significant $(P<0 \cdot 05)$. Multiple testing was addressed using the $P$-value adjustment method that controls the false discovery rate ${ }^{(16)}$. SAS version 9.2 (SAS Institute Inc.) was used for the analysis.

\section{Results}

Male sex, high maternal education level and parental allergic history were associated with an increased risk of CMA in offspring (Table 1). On the other hand, maternal smoking and having pets indoors during the 1 st year of life were associated 
Table 1. Distribution of cows' milk allergy by background characteristics in the Finnish Type 1 Diabetes Prediction and Prevention (DIPP) nutrition study (Numbers and percentages; $n$ 6288)

\begin{tabular}{|c|c|c|c|c|c|}
\hline & \multirow{2}{*}{\multicolumn{2}{|c|}{ Distribution }} & \multicolumn{3}{|c|}{ Cows' milk allergy* } \\
\hline & & & \multicolumn{2}{|c|}{ Cases } & \multirow[b]{2}{*}{$P \dagger$} \\
\hline & $\%$ & $n$ & $\%$ & $n$ & \\
\hline Sex & & & & & $<0.001$ \\
\hline Boys & $52 \cdot 5$ & 3304 & $9 \cdot 8$ & 324 & \\
\hline Girls & 47.5 & 2984 & 6.9 & 205 & \\
\hline Season of birth & & & & & 0.360 \\
\hline Spring (April-May) & $18 \cdot 2$ & 1142 & 7.5 & 86 & \\
\hline Summer (June-August) & $27 \cdot 2$ & 1709 & 7.9 & 135 & \\
\hline Autumn (September-November) & $22 \cdot 2$ & 1394 & 8.8 & 122 & \\
\hline Winter (December-March) & 32.5 & 2043 & $9 \cdot 1$ & 186 & \\
\hline Age of the mother at delivery (years) & & & & & 0.002 \\
\hline$<25$ & $19 \cdot 4$ & 1221 & $5 \cdot 8$ & 71 & \\
\hline $25-29$ & 33.8 & 2127 & 8.7 & 186 & \\
\hline $30-34$ & $28 \cdot 7$ & 1806 & $9 \cdot 6$ & 174 & \\
\hline$\geq 35$ & $18 \cdot 0$ & 1134 & $8 \cdot 6$ & 98 & \\
\hline Maternal vocational education & & & & & $<0.001$ \\
\hline No professional education & $7 \cdot 7$ & 469 & $5 \cdot 1$ & 24 & \\
\hline Vocational school or course & 28.4 & 1725 & $6 \cdot 3$ & 108 & \\
\hline Upper secondary vocational education & 41.9 & 2541 & 9.7 & 246 & \\
\hline Academic education & $22 \cdot 0$ & 1333 & $10 \cdot 4$ & 138 & \\
\hline Missing information & & 220 & & 13 & \\
\hline Maternal smoking status during pregnancy & & & & & 0.009 \\
\hline No & 89.8 & 5449 & $8 \cdot 7$ & 476 & \\
\hline Yes & $10 \cdot 2$ & 620 & 5.7 & 35 & \\
\hline Missing information & 3.5 & 219 & $8 \cdot 2$ & 18 & \\
\hline Urbanity of the place of living & & & & & 0.045 \\
\hline Rural & $13 \cdot 3$ & 831 & 6.5 & 54 & \\
\hline Semi-urban & 9.7 & 606 & $10 \cdot 1$ & 61 & \\
\hline Urban & 77.0 & 4800 & 8.5 & 410 & \\
\hline Missing information & & 51 & & 4 & \\
\hline Maternal asthma or allergic rhinitis & & & & & $<0.001$ \\
\hline No & $54 \cdot 7$ & 1544 & $7 \cdot 8$ & 120 & \\
\hline Yes & $45 \cdot 3$ & 1278 & $12 \cdot 1$ & 155 & \\
\hline Missing information & & 3469 & & 254 & \\
\hline Paternal asthma or allergic rhinitis & & & & & $<0.001$ \\
\hline No & $60 \cdot 0$ & 1659 & 8.0 & 133 & \\
\hline Yes & $40 \cdot 0$ & 1104 & $12 \cdot 2$ & 135 & \\
\hline Missing information & & 3525 & & 261 & \\
\hline Pets inside home during the 1 st year of life & & & & & 0.006 \\
\hline No & 68.5 & 1991 & $10 \cdot 7$ & 213 & \\
\hline Yes & 31.5 & 917 & $7 \cdot 4$ & 68 & \\
\hline Missing information & & 3380 & & 248 & \\
\hline Maternal use of vitamin D supplements during pregnancy (including multivitamin supplements) & & & & & 0.003 \\
\hline No & $67 \cdot 2$ & 3307 & $8 \cdot 2$ & 272 & \\
\hline Yes & $32 \cdot 8$ & 1614 & $10 \cdot 9$ & 176 & \\
\hline Missing information & & 1367 & & 81 & \\
\hline Maternal use of folic acid supplements during pregnancy (including multivitamin supplements) & & & & & $<0.001$ \\
\hline No & $64 \cdot 0$ & 3151 & $8 \cdot 1$ & 254 & \\
\hline Yes & $36 \cdot 0$ & 1770 & $11 \cdot 0$ & 194 & \\
\hline Missing information & & 1367 & & 81 & \\
\hline Maternal use of vitamin D supplements during lactation (including multivitamin supplements) & & & & & 0.004 \\
\hline No & $64 \cdot 7$ & 1901 & 8.6 & 164 & \\
\hline Yes & $35 \cdot 3$ & 1039 & 11.9 & 124 & \\
\hline Missing information & & 3348 & & 241 & \\
\hline Maternal use of folic acid supplements during lactation (including multivitamin supplements) & & & & & 0.006 \\
\hline No & $68 \cdot 1$ & 2001 & 8.8 & 175 & \\
\hline Yes & 31.9 & 939 & $12 \cdot 0$ & 113 & \\
\hline Missing information & & 3348 & & 241 & \\
\hline
\end{tabular}

* Cumulative incidence of cows' milk allergy by the age of 3 years.

† Comparison with $\chi^{2}$ or two-sided Fisher's exact test comparing distributions of cows' milk allergy across categories.

with a lower risk of CMA. The cumulative incidence of CMA was $8.4 \%$ by the age of 3 years. About the same proportion, one-third of the mothers, received folic acid and vitamin D from supplements both during pregnancy and lactation (Table 1). Most of them received both supplements: during pregnancy $26.7 \%$ and during lactation $29.3 \%$ of the mothers had used both 
folic acid and vitamin D supplements. Energy intake reduced from pregnancy to lactation, and the quality of the diet, as assessed by the energy-adjusted intakes of the studied nutrients from food, was poorer during lactation (Table 2).

Maternal dietary supplement use was associated with risk of CMA: children had a higher risk of CMA if their mothers had used folic acid or vitamin D supplementation during pregnancy or lactation (users $v$. non-users; Table 1 ). After adjusting for potential confounding factors, only vitamin D supplementation remained significantly associated with increased risk of CMA (Table 3). Higher folate intake by the mother both during pregnancy and lactation was associated with an increased risk of CMA in the offspring (Table 3). Vitamin D intake from food during pregnancy was not associated with the risk of CMA in the crude analysis, but the association was statistically significant after adjusting for background factors $(P=0.033$ in the first adjustment and $P=0.048$ in the second adjustment)
(Table 3). Adjusting dietary intake during lactation for the intake during pregnancy did not change the results (data not shown). After controlling for the false discovery rate, only the unadjusted findings of an association between folic acid supplement use during pregnancy and increased risk of CMA remained significant.

Stratifying according to maternal history of asthma or allergic rhinitis revealed that higher maternal intake of folic acid was associated with a higher risk of CMA only in children of allergic mothers (OR 1.34; CI 1.14, 1.57, $P=0.007$ for allergic and OR 0.89; CI $0.69,1.16$ for non-allergic mothers; $P=0.008$ for interaction).

Allergic mothers differed from non-allergic mothers in some respects: they were younger and they had used folic acid and vitamin D supplements more often during lactation (online Supplementary Table S1). The amount of folic acid and vitamin $\mathrm{D}$ intake from dietary supplements was higher for

Table 2. Maternal daily intake of energy and nutrients during pregnancy and lactation (Mean values and standard deviations)

\begin{tabular}{|c|c|c|c|c|c|c|c|}
\hline & \multicolumn{2}{|c|}{ During pregnancy (n 4861) } & \multicolumn{2}{|c|}{ During lactation (n 2915) } & \multirow[b]{2}{*}{$P^{\star}$} & \multicolumn{2}{|c|}{ Reference value ${ }^{(39)}$} \\
\hline & Mean & SD & Mean & SD & & Pregnancy & Lactation \\
\hline Energy (kJ) & 11430 & 3370 & 9956 & 2884 & $<0.001$ & & \\
\hline Protein (energy \%) & $16 \cdot 4$ & $2 \cdot 2$ & $16 \cdot 7$ & $2 \cdot 3$ & $<0.001$ & $10-20$ & $10-20$ \\
\hline Carbohydrate (energy \%) & $49 \cdot 6$ & $5 \cdot 1$ & $48 \cdot 7$ & $5 \cdot 6$ & $<0.001$ & $45-60$ & $45-60$ \\
\hline Fat (energy \%) & $33 \cdot 8$ & $4 \cdot 8$ & $34 \cdot 3$ & $5 \cdot 0$ & $<0.001$ & $25-40$ & $25-40$ \\
\hline Vitamin $D$ total $(\mu \mathrm{g})$ & 6.9 & $4 \cdot 0$ & $7 \cdot 3$ & $5 \cdot 0$ & $<0.001$ & 10 & 10 \\
\hline Vitamin D diet $(\mu \mathrm{g})$ & 5.5 & 2.9 & $5 \cdot 0$ & 2.7 & $<0.001$ & & \\
\hline Vitamin D supplements $(\mu \mathrm{g})$ & 1.4 & $2 \cdot 6$ & $2 \cdot 3$ & 4.0 & $<0.001$ & & \\
\hline Folate + folic acid total $(\mu \mathrm{g})$ & $409 \cdot 8$ & $154 \cdot 0$ & 359.5 & $157 \cdot 4$ & $<0.001$ & 500 & 500 \\
\hline Folate diet $(\mu \mathrm{g})$ & 364.0 & $120 \cdot 5$ & $302 \cdot 0$ & $101 \cdot 3$ & $<0.001$ & & \\
\hline Folic acid supplements $(\mu \mathrm{g})$ & $45 \cdot 8$ & 93.0 & 57.5 & $120 \cdot 2$ & $<0.001$ & & \\
\hline
\end{tabular}

* For the pairwise comparison, information on maternal nutrient intake during both pregnancy and lactation was available for 2838 mothers. Pairwise comparison for difference between energy-adjusted intakes during pregnancy and lactation was carried out using Wilcoxon's signed-rank test.

Table 3. Associations between maternal daily intake of nutrients during pregnancy and lactation with the risk of cows' milk allergy in the offspring by 3 years of age

(Odds ratios and $95 \%$ confidence intervals)

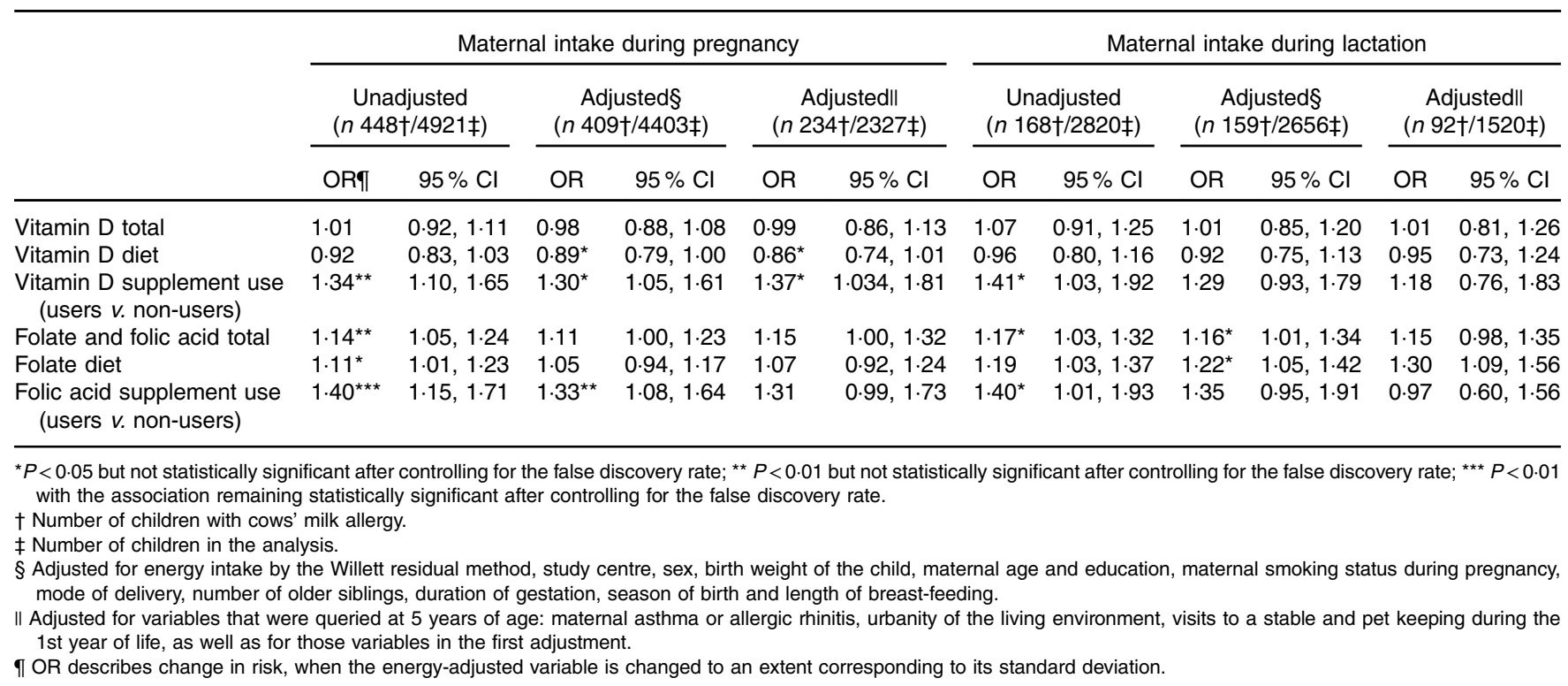


allergic mothers compared with non-allergic mothers both during pregnancy and lactation. Otherwise the diets did not differ between these groups (online Supplementary Table S2).

There were some differences between children who participated in the allergy study and those who declined to participate or had dropped out of the study by the age of 5 years when the allergy study was performed. Participating children compared with non-participants had older, more educated and less frequently smoking mothers who had used more often folic acid and vitamin D supplements (data not shown). Moreover, the amount of folic acid supplementation during pregnancy and lactation was higher among mothers of participating than non-participating children, whereas no difference was seen in the amount of vitamin D supplementation (data not shown). No differences according to participation were observed in maternal energy or energy-yielding nutrient intakes or in folate or vitamin D intake from food (data not shown).

\section{Discussion}

The results of this study suggest that maternal nutrient intake during pregnancy and lactation is associated with the development of CMA in offspring. High maternal intake of folate appears to be associated with a higher risk of CMA in the offspring. On the other hand, maternal intake of vitamin D from food during pregnancy seems to be associated with a decreased risk of CMA in the offspring. Then again, supplemental intakes of both folic acid and vitamin D were associated with an increased risk of CMA. The association between dietary supplement use and CMA risk can at least partly be explained by increased health-seeking behaviour among more educated mothers who also use more dietary supplements. However, after considering multiple comparisons by using the false discovery rate, these associations became marginal.

To our knowledge, this is the first study to examine the associations between maternal folate, folic acid and vitamin D intake during pregnancy and lactation and subsequent CMA in offspring.

A major strength of our study is that data were collected prospectively from a large number of mothers and children. In addition, a major advantage is the good quality of dietary information, which was gathered by a detailed, validated FFQ. In the validation of the FFQ, conducted by Erkkola et al. ${ }^{(6)}$, Pearson's correlations with food records were 0.48 for folic acid and 0.44 for vitamin D. Cross-classification in quintiles revealed decent associations for both nutrients.

Collection of dietary information with a FFQ also comes with some limitations: it is subject to recall bias as well as having the tendency to overestimate food intake. Despite these shortcomings, the aforementioned validation study showed the method to be valid in studying maternal dietary intakes during pregnancy and lactation. The exactly same setting was used in the present study ${ }^{(6)}$.

A limitation of the present study is also that the subjects carried HLA-conferred genetic susceptibility to type 1 diabetes, representing about $15 \%$ of all newborn infants in Finland. An increased intestinal permeability in those with type 1 diabetes or genetic disease susceptibility has been proposed ${ }^{(17)}$. This may limit the generalisability of our results to the overall population, although a similar incidence of CMA has been reported in a genetically unselected Finnish cohort of children ${ }^{(18)}$.

Although being in the same magnitude with other birth cohort studies, dropouts may have led to bias. Children who participated in the allergy study at the age of 5 years had older and more highly educated mothers, who were more often non-smokers, than those who did not participate. These factors are also associated with the child's risk of CMA, which could potentially confound the results. The study period expanding over several years could be a limitation; however, the diagnostic criteria of CMA have remained the same throughout the study years.

We took several possible confounders into the statistical model in order to specifically evaluate the effects of the selected nutrients and to take into account putative confounding by some lifestyle factors associated with the intake of these nutrients. Nutrient intake depends not only on diet but also on the use of dietary supplements; we found that both vitamin D and folic acid supplement use by mothers was associated with an increased risk of CMA in the offspring in this cohort. Supplement users were more highly educated, health conscious and smoked less than non-users, which also may confound the results $^{(19)}$. Maternal education level also correlated with the risk of CMA in the offspring, possibly at least partly explaining the findings, because highly educated mothers may be more prone to seek for a CMA diagnosis for their children. Maternal education level was adjusted for in the analysis to reduce this confounding. Unfortunately, the diet of the children themselves could not be controlled for. A further limitation is that the age of onset of symptoms of CMA was not known, and only the age at diagnosis was known, although these two should coincide. When studying the association between maternal diet during lactation and CMA in the offspring, those whose CMA diagnosis was confirmed before completing the FFQ during lactation were excluded from the analysis in an attempt to rule out reverse causation.

Our findings on background factors and risk of CMA are in line with a previous, large, register-based study, which found that maternal smoking, younger age and low socio-economic status of the mother were associated with a lower risk of $\mathrm{CMA}^{(20)}$. We do not believe that smoking would in fact decrease the risk of CMA; it is assumed that smoking is a surrogate for other environmental factors or may be related to lower levels of health-seeking behaviour. The lower risk of CMA with pet keeping, on the other hand, follows the principles of the hygiene hypothesis and is in line with previous reports on the aetiology of food allergy ${ }^{(21)}$.

Evidence for an association between maternal dietary intake of vitamin D during pregnancy and lactation and atopic outcomes is equivocal. Gale et al. and Weisse et al. have reported a higher risk of eczema, asthma, food allergy or sensitisation against food allergens in children with high maternal 25-OH-vitamin D levels ${ }^{(22,23)}$, whereas Camargo et $a l^{(24)}$ found an inverse association between maternal vitamin D intake and asthma, but not with eczema. Furthermore, Devereux et al. ${ }^{(25)}$ found no association between maternal vitamin D intake during pregnancy with risk of atopic 
sensitisation or asthma in children. In addition, two previous reports from our cohort ${ }^{(26,27)}$ have found an inverse association between maternal vitamin $\mathrm{D}$ intake during pregnancy and allergic outcomes. From our cohort, we have reported a lower risk of asthma and allergic rhinitis by the age of 5 years with high maternal intake of vitamin D during pregnancy ${ }^{(26)}$ as well as a lower risk of atopic sensitisation, measured by specific IgE, to any of the most common food allergens ${ }^{(27)}$. Meta-analysis of observational birth cohort studies showed that increasing intake of dietary vitamin $\mathrm{D}$ during pregnancy was associated with a reduced risk of asthma symptoms in the offspring ${ }^{(28)}$

Recently, two trials showed that prenatal vitamin D supplementation with a daily dose of 60 and $100 \mu \mathrm{g}$, respectively, as compared with the $10 \mu \mathrm{g}$ usual care, did not significantly affect the absolute incidence of asthma-like symptoms ${ }^{(29,30)}$. However, these trials evaluated the effect of additional high dosage of vitamin D on top of usual care, whereas the observational studies have compared sub-optimal $v$. optimal intake in relation to dietary recommendations. In the present study, higher maternal intake of vitamin $\mathrm{D}$ from food tended to be associated with a lower risk of CMA in offspring, but the association was not consistent after all the adjustments.

Adding to the confusion, in the present study, vitamin D intake from supplements was associated with a higher risk of CMA in offspring. It seems unlikely that the small amount of vitamin D derived from supplements would actually be harmful, and the association is probably explained by other lifestyle factors. This association between supplement use and risk of CMA is likely to dilute the potential real association between vitamin $\mathrm{D}$ intake from food and CMA risk. As a limitation, sunlight exposure as a source of vitamin D or 25-OH-vitamin D levels was not studied. However, there was no variation in CMA incidence by birth season (Table 1), suggesting that the influence of sunlight exposure on CMA development is inconsequential. Potential mechanisms on how vitamin D deficiency could contribute to the development of food allergy include increased $\mathrm{T}$ cell proliferative responses, IL leading to Th1-type reactions as well as decreasing tolerogenic dendritic cells, regulatory T cell production and IL-10, in addition to compromising intestinal barrier function (recently reviewed by Vassallo et l. $^{(31)}$ ).

Folate metabolism is important in DNA methylation, and has therefore been implicated to play a role in epigenetics ${ }^{(32)}$. A recent meta-analysis found no evidence of association between folic acid supplementation in the first trimester and risk of asthma in offspring ${ }^{(33)}$. One cohort looking at maternal folate intake during the first trimester did not find associations with risk of eczema $^{(34)}$, whereas another cohort did not find associations between maternal intake of folate or folic acid supplements during the last trimester and risk of atopy in offspring ${ }^{(35)}$. However, another report found that not dietary folate intake but supplementation with folic acid above $500 \mu \mathrm{g} / \mathrm{d}$ to be associated with the development of eczema in children ${ }^{(36)}$. Although the association between maternal folate and folic acid intake and risk of CMA in offspring found in our study is only modest and the cohort design cannot rule out all the confounding factors, we cannot rule out the potential real association either.

There is a strong hereditary component in allergic diseases. The immunological environment in utero is determined by the immunology of the mother and the interaction between the mother and the fetus. It is possible that the immunological effects of nutrients differ in allergic individuals from those without atopic diseases. Therefore, we examined the associations between nutrients and CMA in children separately with allergic and nonallergic mothers. The associations did, in fact, differ somewhat: maternal intake of folic acid during pregnancy was associated with a higher risk of CMA in offspring of allergic mothers only. Granell et $a l^{(35)}$ assessed a genetic polymorphism in gene MTHFR C677T and its association with atopic disease; their study did not support the hypothesis that impaired folate metabolism would be associated with allergy. They did, however, discover that folic acid supplementation by mothers during pregnancy was related to an increased risk of atopy in children carrying a certain $\mathrm{T}$ allele, which has previously been linked to an increased risk of allergic disease ${ }^{(37)}$. Further studies are needed to explore the immunological mechanisms behind this finding.

Mothers who use dietary supplements differ in other aspects of lifestyle from those who do not use supplements: they have higher educational levels and smoke less ${ }^{(38)}$. It is possible that those with an overall healthier lifestyle may be more prone to seek medical assistance, thus increasing the likelihood of receiving a CMA diagnosis for their child, which could confound the associations observed in this study. Even though the associations prevailed after adjusting for maternal level of education, it is possible that there remains residual confounding and the association between supplemental folic acid and vitamin D intake and higher risk of CMA in children that we found is not causal.

Average folate, folic acid and vitamin D intakes from food and supplements of the mothers during pregnancy and lactation were below the recommended 10 and $500 \mu \mathrm{g} / \mathrm{d}$, respectively ${ }^{(39)}$. As a substantial amount of these vitamins is derived from supplements, it is not feasible to adjust for supplement use in the regression model. A majority of the mothers used both folic acid and vitamin D supplements, making it difficult to differentiate between their separate associations with CMA risk. In the end, we cannot be certain whether the association between supplemental intake and the risk of CMA is causal or whether it acts as a proxy for some other lifestyle factor/s.

In conclusion, these results are the first to link maternal folate, folic acid and vitamin D intakes during pregnancy and lactation with the development of CMA in offspring. The associations found are marginal and need to be confirmed or rejected in further studies. The association between maternal intake of folic acid and CMA in offspring calls for precaution in the use of supplements. However, the slightly higher risk of CMA with high intake of folic acid does not warrant changes in recommending higher intake of folic acid during pregnancy to prevent conditions such as neural tube defects. Adhering to the dietary guidelines for pregnant and lactating women leads to sufficient, balanced intake of nutrients; excessive intake of nutrients is not advised, as it may have adverse immunological effects.

\section{Acknowledgements}

The authors are extremely grateful to all the families who took part in this study. The authors also acknowledge the excellent 
collaboration of the DIPP research nurses, doctors, nutritionists and laboratory staff over the years.

The study was supported by the Academy of Finland (grants 63672, 79685, 79686, 80846, 201988, 210632, 129492, 126813), the Finnish Paediatric Research Foundation, the Juho Vainio Foundation, the Yrjö Jahnsson Foundation, the Competitive Research Funding of the Tampere University Hospital, Medical Research Funds of Turku and Oulu University Hospitals, the European Foundation for the Study of Diabetes, the Juvenile Diabetes Research Foundation (grants 197032, 4-1998-274, 4-1999-731, 4-2001-435), the Novo Nordisk Foundation and EU Biomed 2 (BMH4-CT98-3314), Doctoral Programs for Public Health, Foundation for Allergy Research, Research Foundation of Orion Corporation, Tampere Tuberculosis Foundation and the Jalmari and Rauha Ahokas Foundation. None of the funders had any role in the design, analysis or writing of this article.

The authors' contributions were as follows: S. M. V., J. T. and M. Kaila were responsible for the current study concept and design; J. I., M. Knip and O. S. designed the DIPP study; S. M. V. designed the nutrition study within DIPP; J. T., S. M. V. and P. L. conducted research; L. J. V. provided registry data from the Social Insurance Institute; H.-M. T. was responsible for the statistical analysis; R. V. was responsible for the clinical work in Oulu and M. Knip for the clinical work in Tampere; J. T. wrote the first version of the manuscript; P. L., M. Kaila and S. M. V. participated in the writing process; S. M. V. had primary responsibility for final content; and all the authors contributed to the critical revision of the manuscript.

The authors declare that there are no conflicts of interest.

\section{Supplementary material}

For supplementary material/s referred to in this article, please visit http://dx.doi.org/doi:10.1017/S0007114516002464

\section{References}

1. Prescott SL (2010) Allergic disease: understanding how in utero events set the scene. Proc Nutr Soc 69, 366-372.

2. Hollingsworth JW, Maruoka S, Boon $\mathrm{K}$, et al. (2008) In utero supplementation with methyl donors enhances allergic airway disease in mice. J Clin Invest 118, 3462-3469.

3. Bozzetto S, Carraro S, Giordano G, et al. (2012) Asthma, allergy and respiratory infections: the vitamin $\mathrm{D}$ hypothesis. Allergy 67, 10-17.

4. Tuokkola J, Luukkainen P, Tapanainen H, et al. (2016) Maternal diet during pregnancy and lactation and cow's milk allergy in offspring. Eur J Clin Nutr 70, 554-559.

5. Kupila A, Muona P, Simell T, et al. (2001) Feasibility of genetic and immunological prediction of type I diabetes in a population-based birth cohort. Diabetologia $\mathbf{4 4}$, 290-297.

6. Erkkola M, Karppinen M, Javanainen J, et al. (2001) Validity and reproducibility of a food frequency questionnaire for pregnant Finnish women. Am J Epidemiol 154, 466-476.

7. National Institute for Health and Welfare, Nutrition Unit (2009) National Institute for Health and Welfare (2009) Fineli ${ }^{\circledR}$ Finnish Food Composition Database. http://www.fineli.fi (accessed December 2015).

8. Prasad M, Lumia M, Erkkola M, et al. (2010) Diet composition of pregnant Finnish women: changes over time and across seasons. Public Health Nutr 13, 939-946.
9. National Public Health Institute (1998) The 1997 Dietary Survey of Finnish Adults. Helsinki: Hakapaino Oy.

10. Männisto S, Ovaskainen M \& Valsta L (2003) The National Findiet 2002 Study. Helsinki: Hakapaino Oy.

11. Tuokkola J, Kaila M, Pietinen P, et al. (2008) Agreement between parental reports and patient records in food allergies among infants and young children in Finland. J Eval Clin Pract 14, 984-989.

12. Tuokkola J, Luukkainen P, Kaila M, et al. (2010) Validation of a questionnaire on cow's milk allergy: parental reports and physician's diagnosis. Acta Paediatr 99, 1273-1275.

13. Nwaru BI, Lumia M, Kaila M, et al. (2011) Validation of the Finnish ISAAC questionnaire on asthma against anti-asthmatic medication reimbursement database in 5-year-old children. Clin Respir J 5, 211-218.

14. Liang KY \& Zeger SL (1986) Longitudinal data analysis using generalized linear models. Biometrica 73, 13-22.

15. Willett W (1998) Nutritional Epidemiology, 2nd ed. New York, NY: Oxford University Press.

16. Benjamini Y \& Hochberg Y (1995) Controlling the false discovery rate: a practical and powerful approach to multiple testing. J R Stat Soc B 57, 289-300.

17. Vaarala O, Atkinson MA \& Neu J (2008) The "perfect storm" for type 1 diabetes: the complex interplay between intestinal microbiota, gut permeability, and mucosal immunity. Diabetes 57, 2555-2562.

18. Pyrhönen K, Näyhä S, Kaila M, et al. (2009) Occurrence of parent-reported food hypersensitivities and food allergies among children aged 1-4 yr. Pediatr Allergy Immunol 20, 328-338.

19. Dickinson A \& Mackay D (2014) Health habits and other characteristics of dietary supplement users: a review. Nutr $J$ 13, 14 .

20. Metsälä J (2010) Maternal and perinatal characteristics and the risk of cow's milk allergy in infants up to two years of age - a case-control study nested in the Finnish population. Am J Epidemiol 171, 1310-1316.

21. Marrs T, Bruce KD, Logan K, et al. (2013) Is there an association between microbial exposure and food allergy? A systematic review. Pediatr Allergy Immunol 24, 311-320.

22. Weisse K, Winkler S, Hirche F, et al. (2013) Maternal and newborn vitamin $\mathrm{D}$ status and its impact on food allergy development in the German LINA cohort study. Allergy $\mathbf{6 8}$, 220-228.

23. Gale CR, Robinson SM, Harvey NC, et al. (2008) Maternal vitamin D status during pregnancy and child outcomes. Eur J Clin Nutr 62, 68-77.

24. Camargo CA Jr, Rifas-Shiman SL, Litonjua AA, et al. (2007) Maternal intake of vitamin D during pregnancy and risk of recurrent wheeze in children at 3 y of age. Am J Clin Nutr 85 , $788-795$.

25. Devereux G, Litonjua AA, Turner SW, et al. (2007) Maternal vitamin $\mathrm{D}$ intake during pregnancy and early childhood wheezing. Am J Clin Nutr 85, 853-859.

26. Erkkola M, Kaila M, Nwaru BI, et al. (2009) Maternal vitamin D intake during pregnancy is inversely associated with asthma and allergic rhinitis in 5-year-old children. Clin Exp Allergy 39, 875-882.

27. Nwaru BI, Ahonen S, Kaila M, et al. (2009) Maternal diet during pregnancy and allergic sensitization in the offspring by 5 yrs of age: a prospective cohort study. Pediatr Allergy Immunol 21, 29-37.

28. Nurmatov U, Devereux G \& Sheikh A (2011) Nutrients and foods for the primary prevention of asthma and allergy: systematic review and meta-analysis. J Allergy Clin Immunol 127, 724-733. 
29. Litonjua AA, Carey VJ, Laranjo N, et al. (2016) Effect of prenatal supplementation with vitamin D on asthma or recurrent wheezing in offspring by age 3 years: the VDAART randomized clinical trial. JAMA 315, 362-370.

30. Chawes BL, Bonnelykke K, Stokholm J, et al. (2016) Effect of vitamin $\mathrm{D}_{3}$ supplementation during pregnancy on risk of persistent wheeze in the offspring: a randomized clinical trial. JAMA 315, 353-361.

31. Vassallo MF \& Camargo CA Jr (2010) Potential mechanisms for the hypothesized link between sunshine, vitamin D, and food allergy in children. J Allergy Clin Immunol 126, 217-222.

32. Miles EA \& Calder PC (2015) Maternal diet and its influence on the development of allergic disease. Clin Exp Allergy 45, 63-74.

33. Crider KS, Cordero AM, Qi YP, et al. (2013) Prenatal folic acid and risk of asthma in children: a systematic review and meta-analysis. Am J Clin Nutr 98, 1272-1281.

34. Miyake Y, Sasaki S, Tanaka K, et al. (2011) Maternal B vitamin intake during pregnancy and wheeze and eczema in Japanese infants aged 16-24 months: the Osaka Maternal and Child Health study. Pediatr Allergy Immunol 22, 69-74.
35. Granell R, Heron J, Lewis S, et al. (2008) The association between mother and child MTHFR C677T polymorphisms, dietary folate intake and childhood atopy in a population-based, longitudinal birth cohort. Clin Exp Allergy 38, 320-328.

36. Dunstan JA, West C, McCarthy S, et al. (2012) The relationship between maternal folate status in pregnancy, cord blood folate levels, and allergic outcomes in early childhood. Allergy $\mathbf{6 7}$, 50-57.

37. Husemoen LL, Toft U, Fenger M, et al. (2006) The association between atopy and factors influencing folate metabolism: is low folate status causally related to the development of atopy? Int J Epidemiol 35, 954-961.

38. Uusitalo L, Uusitalo U, Ovaskainen ML, et al. (2008) Sociodemographic and lifestyle characteristics are associated with antioxidant intake and the consumption of their dietary sources during pregnancy. Public Health Nutr 11, 1379-1388.

39. Nordic Council of Ministers (2012) Nordic Nutrition Recommendations 2012. http://dx.doi.org/10.6027/Nord2014-002 (accessed December 2015). 